International Journal of Pharmaceutical Sciences and Drug Research 2020; 12(1): 46-52

Contents lists available at UGC-CARE
International Journal of Pharmaceutical
Sciences and Drug Research
[ISSN: 0975-248X; CODEN (USA): IJPSPP]

Research Article

\title{
In silico Screening of ZINC Database for Discovery of Novel Urease Inhibitors as a Remedy to Gastro-duodenal Ulcer Caused by Helicobacter pylori
}

\author{
K. S. Chopdar ${ }^{1}$, P. K. Mohapatra ${ }^{2}$, B. Nayak ${ }^{3}$, M. K. Raval ${ }^{4 *}$ \\ ${ }^{1}$ Department of Zoology, Rajendra College, Balangir-767002, Odisha, India \\ ${ }^{2}$ Department of Chemistry, CV Raman College of Engineering, Bhubaneswar-752054, Odisha, India \\ ${ }^{3}$ School of Life Sciences, Sambalpur University, Sambalpur-768019, Odisha, India \\ ${ }^{4}$ Department of Chemistry, Gangadhar Meher University, Sambalpur-768004, Odisha, India
}

\begin{tabular}{l} 
A R T I C L E I N F O \\
\hline Article history: \\
Received: 21 October, 2019 \\
Revised: 31 December, 2019 \\
Accepted: 01 January, 2020 \\
Published: 30 January, 2020 \\
Keywords: \\
Covalent inhibitor, Gastro- \\
duodenal ulcer, Helicobacter \\
pylori, Structure-based drug \\
design, Urease inhibitors. \\
D0I: \\
10.25004/IJPSDR.2020.120108
\end{tabular}

\section{INTRODUCTION}

Helicobacter pylori (H. pylori) is known to form a colony in the human stomach inducing gastro-duodenal ulcer. ${ }^{[1-2]}$ H. pylori is considered as the most common infectious agent related to stomach cancer. ${ }^{[3]}$ The chronic inflammation of normal gastric mucosa by the bacterium leads to atrophic gastritis, which subsequently leads to intestinal dysplasia and metaplasia. The condition further worsens to the development of carcinoma. ${ }^{[4]}$ The bacterium is capable of surviving and growing in the acidic medium of the stomach because of the production of ammonia as a result of catalytic hydrolysis of urea by urease. ${ }^{[5-6]}$ The catalytic reaction involving hydrolysis of urea has been proposed as follows. ${ }^{[7-9]}$

\begin{abstract}
A B S T R A C T
Design and synthesis of novel urease inhibitors taking center stage nowadays with specific attention as a remedy to Helicobacter pylori infection. Several inhibitors fail in-vivo and clinical trials owing to the toxicity and hydrolytic profile. In the present study, we are making an attempt to screen a large small-molecule database, ZINC, for a potential urease inhibitor. The structure-based drug discovery approach has been adopted with acceptable absorption distribution metabolism excretion (ADMET) parameters so that the lead molecules may have fair chances of passing in vitro and in-vivo trials. The lead molecule in our study, with ID ZINC90446454, is a urea derivative and predicted to be nontoxic. It comes out to be a promising drug candidate with pKd value 7.83, LE 0.429 , and $\mathrm{LD}_{50}$ value $10100 \mathrm{mg} / \mathrm{kg}$ body weight. Its sulfanyl derivative, with predicted high $\mathrm{LD}_{50}(10100 \mathrm{mg} / \mathrm{kg}$ body weight), exhibits the feasibility of a disulfide high specificity, high potency and low toxicity. The derivative, in the future, may be a successful drug candidate for H. pylori-induced gastro-duodenal ulcer.
\end{abstract}

\footnotetext{
*Corresponding Author: Dr. Mukesh Kumar Raval

Address: Department of Chemistry, Gangadhar Meher University, Sambalpur-768004, Odisha, India

Email $\bowtie:$ mraval@yahoo.com

Tel.: +91-9437110137

Relevant conflicts of interest/financial disclosures: The authors declare that the research was conducted in the absence of any commercial or financial relationships that could be construed as a potential conflict of interest.

Copyright (C) 2020 K. S. Chopdar et al. This is an open access article distributed under the terms of the Creative Commons Attribution- NonCommercialShareAlike 4.0 International License which allows others to remix, tweak, and build upon the work non-commercially, as long as the author is credited and the new creations are licensed under the identical terms.
} 
suffering more from the infections caused by $H$. pylori. It is also reported that in certain areas of the world about $50 \%$ of the population are infected with $H$. pylori. ${ }^{[5-6]}$ The present regimen for the treatment is a triple therapy which comprises of a proton pump inhibitor along with any two from the following antibiotics amoxicillin (AMX), clarithromycin (CLA), metronidazole (MNZ), and tetracycline (TET). ${ }^{[6]}$ The antibiotic drugs are associated with various side effects, and also the prolonged use of antibiotics develops resistance in the bacterium. The treatment of gastroduodenal ulcers with these drugs has limitations. ${ }^{[11]}$ Therefore, an alternative line of treatment against $H$. pylori is being explored. Urease is a crucial survival factor for the bacterium, is expected to be a potential target for drug development. The absence of urease in an essential function of human is an added advantage in selecting urease as the target. Hence inhibitors of urease may serve as potential anti- $H$. pylori drug candidates. Several small molecules have been reported to be potent inhibitors of the urease. However, many of them have failed as drug candidates due to toxicity or their inefficiency in vivo. Hydrolysis of inhibitors may be a significant factor in its failure in vivo. There is a need to search for molecules that should satisfy the inhibitory property, low toxicity, and sustainability against hydrolysis. In the present work, we have attempted to screen a large small-molecule database, ZINC, for a urease inhibitor with acceptable ADMET parameters so that the lead molecules may have fair chances of passing in vitro and in-vivo trials.

\section{Materials AND Methods}

\section{Selection and Preparation of $\boldsymbol{H}$. pylori Urease}

The coordinate file of $\mathrm{x}$-ray crystallographic structure of H. pylori urease in complex with acetohydroxamic acid with a resolution of $3 \AA$ was obtained from the Protein Data Bank (PDB ID 1E9Y). The enzyme contains two chains, i.e., chain $A$ and chain B. The chain A contains 238 amino acids, and chain B has 569 amino acids. Chain B contains the active site for the binding and hydrolysis of urea. The active site comprises of two $\mathrm{Ni}^{+2}$ separated by a distance of $3.02 \AA$. The two $\mathrm{Ni}^{+2}$ are coordinated by carboxylate bridge of a carbamylated lysine (KCX219). His248 and His274 coordinate the Ni3001. The Ni3002 is coordinated by His136, His138, and Asp362. The acetohydroxamic acid (HAE800) coordinates to both the $\mathrm{Ni}^{+2}$. [12]

The chain A of $1 \mathrm{e} 9 \mathrm{y}$ was deleted from the coordinate file of $1 \mathrm{e} 9 \mathrm{y}$ and saved as $1 \mathrm{e} 9 \mathrm{yB}$. The binding site residues of the active site for HAE ligand were identified by using "make a binding site from the ligand" module of ArgusLab 4.0.1. All water molecules were also removed. The residues identified to be present in the binding site are Asp165, Asn168, His221, Glu222, Asp223, Thr251, Cys321, His322, and Arg338.

\section{Docking with ZINC Database}

The active site of urease (1e9yB.pdb) was docked with ZINC database using i-Dock online docking server. i-Dock uses the algorithm of scoring function of AutoDock Vina. Small molecules from ZINC database ${ }^{[13]}$ with the following filtering parameters were screened by i-Dock: (a) Molecular weight (g/mol): [220- 500] (b) Partition coefficient xlogP: [1-3] (c) Rotatable bonds: [4-6] (d) Hydrogen bond donors: [2-4] (e) Hydrogen bond acceptors: [4-6] (f) Net charge: [0-0] (g) Apolar desolvation (kcal/mol): [0-10] (h) Polar desolvation (kcal/mol): [-40-0] (i) Polar surface area tPSA (Å2): [60-80]. From the huge ZINC database $1,58,417$ compounds pass through the above filter. The receptor was kept rigid along with the binding site residues within a cubic box of $20 \AA$ with center at coordinate $(128,129,87)$, to confine the conformational space to a definite cavity of the receptor. ${ }^{[14]}$ Binding free energy calculation was done as a sum of electrostatic energy, internal energy due to flexible conformational changes, Van der Waals energy, and translational and rotational energy. The more negative the free energy of a ligand-receptor complex, the more stable the complex is. The top 100 ligands were selected from the list. The output from i-Dock is saved as hits.csv and hits.pdbqt. The hits.csv contains binding free energy (iDock score), ligand efficiency, RF-score (binding affinity prediction by random forest method), hydrogen bonds, molecular properties, list of vendors of hit compounds in a tabular format. The hits.pdbqt stores predicted conformations of the hit compounds in pdbqt format.

\section{Lead Identification}

The top 100 hit molecules are subjected for screening to check its LipE and ligand efficiency dependent lipophilicity (LELP) ADMET and drug likeness through FAF drugs4 and FAF QED, toxicity screening through ProToxII, and druglikeness score by MolSosft.

\section{LELP Scoring}

From the result of i-dock score two important parameters were calculated, LipE and LELP, those were used for lead identification. If LLE or LipE scoring is $>3$ and LELP scoring is $<10$ then the molecule is treated as good lead-like. If LipE is $>5$ and LELP is within 0 to 7.5 then the molecule is considered as a good clinical drug-like compound. [15] Parameters used for the calculation of LipE and LELP are:

- Ligand Efficiency (LE): LE is proposed to be a parameter, which compares the average binding energy per atom of the molecules ${ }^{[15]}$

$\mathrm{LE}=(1.37 / \mathrm{HA}) * \mathrm{pIC} 50$ or $\mathrm{LE}=(1.37 / \mathrm{HA}) * \mathrm{pKd}$

where, HA: The number of heavy atoms, pIC50: the negative logarithm to the base 10 of the half-maximal inhibitory concentration, pKd: negative logarithm to the base 10 of dissociation constant.

LipE or LLE: Lipophilic ligand efficiency is calculated as the difference between pIC50 and lipophilicity (xLogP) 
and is an estimate of the specificity of a molecule in binding to the target relative to partitioning into 1-octanol: water. $^{[16]}$

$$
\text { LipE }=\text { pIC50 }-x \log P \text { or LipE }=p K d-x \log P
$$

LELP: it is called as ligand efficiency dependent lipophilicity is defined as the ratio of xlogP and LE. ${ }^{[17]}$

LELP $=x \operatorname{xog} P / L E$

\section{Drug Likeness}

The prediction for drug-likeness of compounds was made by an online tool FAF-drugs4 (Free ADME-TOX filtering tool 4) developed by Molecules Therapeutic in silico (MTI) of University of Paris, France ${ }^{[18]}$ In house Drug-like soft and PAINS A, B, and $\mathrm{C}^{[19,20]}$ filters were selected to predict the properties of the compounds and to categorize them into accepted, intermediate, or rejected.

\section{FAF QED (Quantitative Estimation of Drug Likeness)}

FAF quantitative estimation of drug-likeness (FAF QED) is an online tool for quantitative estimation of the compound to be drug like in a scale $0-1 .{ }^{[21]}$ For the computation of FAF QED eight physicochemical parameters are considered, namely, molecular weight (MW), octanolwater partition coefficient $(\log \mathrm{P})$, the number of hydrogen bond donors and acceptors (HBD and HBA), polar surface area of the molecule (PSA), the number of rotatable bonds (ROTB), the number of aromatic rings (AROM), and already published and known 113 structural alerts (ALERT).

\section{Toxicity Screening Through ProTox-II}

ProTox-II is a virtual lab for the prediction of toxicities of small molecules. ${ }^{[22]}$ It is a freely available web server for computing toxicity of small molecules. It can be accessed free online (http://tox.charite.de/protox_II). The webserver enables screening and prediction of toxicity of a molecule at a broad range of toxicity endpoints. Many models of toxicity prediction are taken into account like oral toxicity, hepatotoxicity, mutagenicity, carcinogenicity, cytotoxicity, and immune-toxicity along with the metabolic pathways which are inhibited by the molecule and more specifically the specific target which is inhibited by the molecule in a toxicological pathway. ${ }^{[22]}$ The toxicity is defined in terms of $\mathrm{LD}_{50}$ value (mg/kg body weight). The $\mathrm{LD}_{50}$ is the oral dose at which death of $50 \%$ of test subjects occurs upon intake of a compound. The $\mathrm{LD}_{50}$ values are classified into the following six classes:

- Fatal $\left(\mathrm{LD}_{50} \leq 5\right)$

- Fatal $\left(5<\mathrm{LD}_{50} \leq 50\right)$

- Toxic $\left(50<\mathrm{LD}_{50} \leq 300\right)$

- Harmful $\left(300<\mathrm{LD}_{50} \leq 2000\right)$

- Probably harmful $\left(2000<\mathrm{LD}_{50} \leq 5000\right)$

- Non-toxic $\left(\mathrm{LD}_{50}>5000\right)$

\section{Lead Optimization}

Lead molecule was structurally modified to obtain lead derivatives (LD) using build module of HyperChem Pro 8.0. The derivatives were geometrically optimized by steepest descent method (1000 step) applying Mm+ force field using HyperChem 8.0.

\section{RESULTS}

\section{Lead Identification}

Top 100 hits in order of idock score were considered for lead identification. The hit molecules were subjected to FAF4 Drugs analysis (supplementary file SF1.xls). All the ligands were found acceptable as a drug like molecules. FAF QED yielded quantitative drug-like property in the scale 0 (most unfavorable) - 1 (most favorable) (supplementary file SF2.xls). The LE, LipE, LELP, ProTox II LD50 and MolSoft Drug-like Scores were estimated (supplementary file SF3.xls) and only ligands with toxicity class 5 and 6 are presented in the Table 1 . These are the leads. The ZINC90446454 was selected for further study as it had the lowest toxicity and the best drug-like score reflected by LipE, LELP, QED and MolSoft score (Table 1).

\section{Lead Optimization}

The lead ZINC90446454 (1-[(1R)-1-benzyl-2-(7-chloro3,4-dihydroisoquinolin-2(1H)-yl)-2-oxoethyl]urea) is a urea derivative (Fig. 1). It is not reported so far as the urease inhibitor as per the best of our knowledge. The interactions of the ligand in the active site of $1 \mathrm{e} 9 \mathrm{yB}$ are depicted in Fig. 2a, b. Cys321, H322, and Arg333 are important interactive residues, which sit in the flap region and monitor inhibition. The $\mathrm{Cl}$ in isoquinoline ring at position 7 in the lead was substituted by Br and $\mathrm{F}$ to obtain the derivatives LD1 and LD2, respectively. It is observed that $\mathrm{H} 12$ at position 4 of the isoquinoline ring is close to

Table 1: Lead molecules selected on the basis of dock score, toxicity, and drug-like score

\begin{tabular}{|c|c|c|c|c|c|c|c|c|c|c|c|c|}
\hline ZINC ID & $\begin{array}{l}\text { Idock } \\
\text { score } \\
\text { (kcal) } \\
\text { mol) }\end{array}$ & $p K d$ & $H A$ & $M W$ & $x \log P$ & $L E$ & LipE & $L E L P$ & $Q E D w$ & $\begin{array}{l}\text { Pro tox } \\
\text { II class }\end{array}$ & $\begin{array}{l}L D_{50} \\
\mathrm{mg} / \mathrm{kg} \\
\text { body } \\
\text { weight }\end{array}$ & $\begin{array}{l}\text { Mol } \\
\text { soft sore }\end{array}$ \\
\hline 47874303 & -8.507 & 6.670 & 24 & 353.756 & 2.75 & 0.381 & 3.920 & 7.223 & 0.885 & 5 & 4640 & 1.47 \\
\hline 70702792 & -8.422 & 6.592 & 25 & 368.277 & 2.81 & 0.361 & 3.782 & 7.779 & 0.831 & 5 & 2250 & -0.34 \\
\hline 65653330 & -8.419 & 7.236 & 26 & 354.385 & 1.99 & 0.381 & 5.246 & 5.219 & 0.751 & 5 & 2200 & 1.06 \\
\hline 90446454 & -8.213 & 7.830 & 25 & 357.841 & 2.56 & 0.429 & 5.270 & 5.966 & 0.882 & 6 & 10100 & 1.01 \\
\hline
\end{tabular}


In silico Screening of ZINC Database<smiles>NC(=O)NC(Cc1ccccc1)C(=O)N1CCc2ccc(Cl)cc2C1</smiles>

Lead ZINC90446454

iDOCK: -8.21

pKd: 7.83<smiles>NC(=O)N[C@@H](Cc1ccccc1)C(=O)N1Cc2ccccc2[C@H](S)C1</smiles>

LD3 iDOCK: -4.82

pKd: 5.01<smiles>CNC(=O)NC[C@H](Cc1ccccc1)C(N)=O</smiles>

LD1 iDOCK: -5.44

pKd: 5.33<smiles>NC(=O)N[C@H](Cc1ccccc1)C(=O)N1Cc2cc(F)ccc2[C@@H](S)C1</smiles>

LD4

iDOCK: -6.90

pKd: 6.87<smiles>CNC(=O)N[C@@H](Cc1ccccc1)C(=O)N1CCc2ccc(F)cc2C1</smiles>

LD2

iDOCK: -7.80

pKd: 6.87<smiles>NC(=O)N[C@@H](Cc1ccccc1)C(=O)N1Cc2cc(Cl)ccc2[C@@H](S)C1</smiles>

LD5

iDOCK: -6.26

pKd: 6.42<smiles>NC(=O)N[C@@H](Cc1ccccc1)C(=O)N1Cc2cc(Br)ccc2[C@@H](S)C1</smiles>

LD6

iDOCK: -6.40

pKd: 6.44

Fig. 1: Structural 2D representation of the lead molecule (ZINC90446454) along with the lead derivatives, LD1 to LD6. iDock score and binding affinity $(\mathrm{pKd})$ values are mentioned below each structure

SG of Cys321 of the enzyme (2.80 ̊). The hydrogen may be substituted with SH group to create an opportunity of forming disulfide linkage between SG of Cys321 and S of the ligand. The covalent ligand binding may improve the inhibitory potency of the ligand. Derivative with SH substitution and $\mathrm{Cl}$ deletion in ZINC90446454 was built 




$\mathrm{b}$
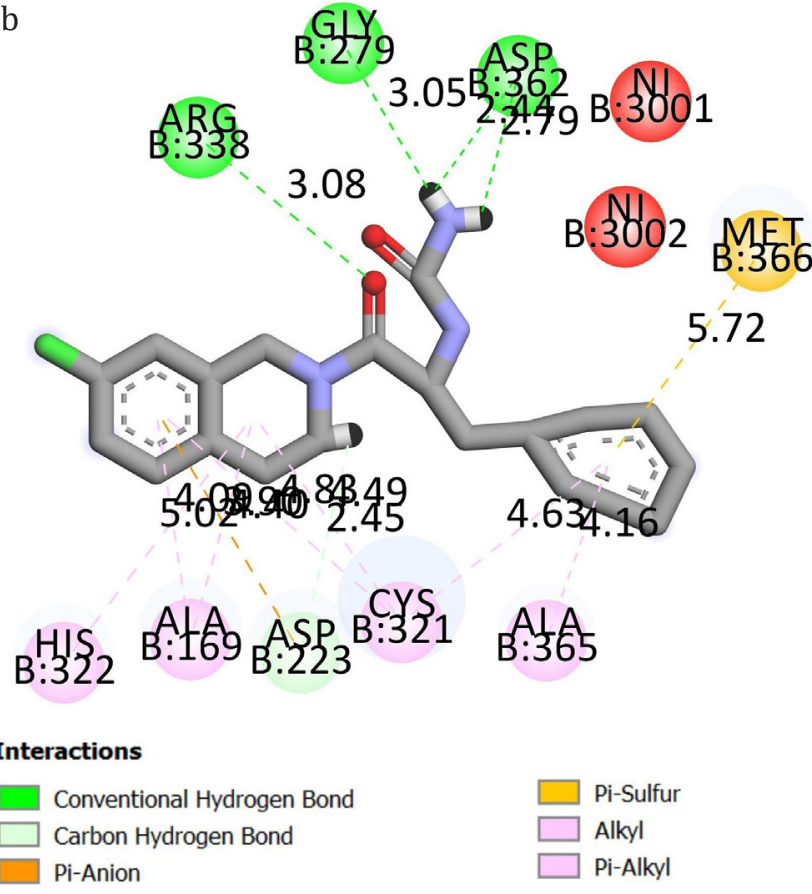

Fig. 2: Interaction of the ligand, ZINC90446454, at the active site of the enzyme $1 \mathrm{e} 9 \mathrm{yB}$, in 3D with atomic details (a) and 2D at residue level only (b). Biovia Discovery Studio 2016 visualizer has been used to prepare the figures.

(LD3). $\mathrm{F}, \mathrm{Cl}$, and $\mathrm{Br}$ substituents in LD3 were built as LD4, LD5 and LD6 respectively (Fig. 1). The iDock score and pKd values are mentioned below each molecular diagram. LD4 (1-\{(1R)-1-benzyl-2-[(4S)-7-fluoro-4-sulfanyl-3,4dihydroisoquinolin-2(1H)-yl]-2-oxoethyl\}urea) may be having the highest potency among all the LDs. The binding interactions of LD4 with active site of enzyme are depicted in Figs. 3 a, b.

\section{The Covalent Ligand Binding}

The covalent binding of the ligand with the receptor was created in silico. The optimized disulfide bond and ligand interactions are presented in Fig. 4a, b. The S-S bond length was measured to be $2.07 \AA$.

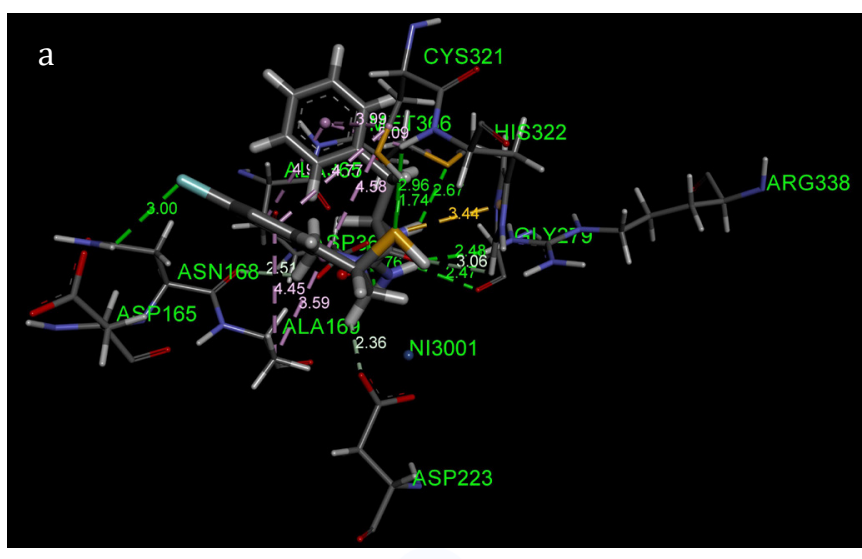

b

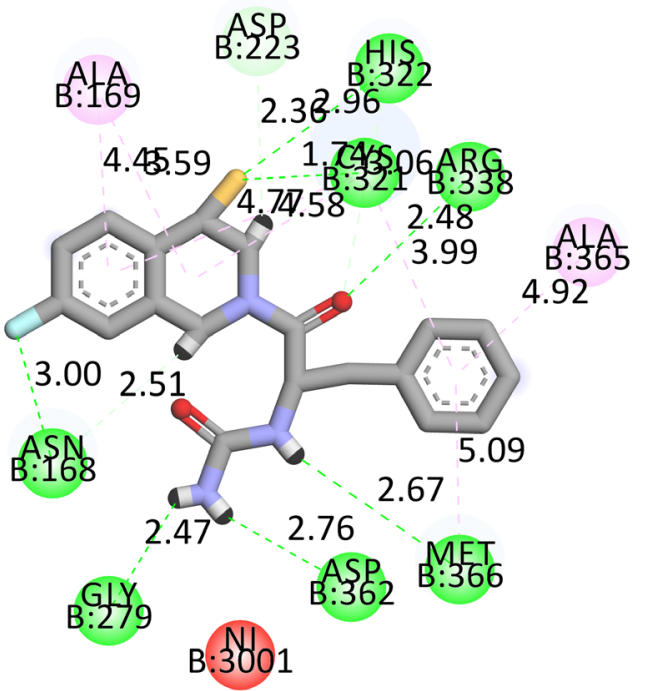

Interactions

Conventional Hydrogen Bond

Carbon Hydrogen Bond

Pi-Sulfur

Fig. 3: Shows interactions of the lead LD4 at the active site of enzyme 1e9yB, in 3D with atomic details (a) and 2D at residue level only (b). Biovia Discovery Studio 2016 visualizer has been used to prepare the figures.

\section{DISCUSSION}

A large number of compounds of different classes have been reported as a urease inhibitor with the potentiality to be used as drug against $H$. pylori. However, equally, a huge number of them are not found suitable as drug candidates due to toxicity and hydrolytic instability during pharmacokinetics. ${ }^{[23]}$ We made an attempt to explore ZINC database containing 3D structures of over 230 million commercially available compounds with an aim to fish an inhibitor of urease with low toxicity and high efficiency.

\section{A Promising Candidate}

The lead molecule ZINC90446454 is a promising drug candidate with value 7.83, LE 0.429 , and $\mathrm{LD}_{50}$ value 10100 $\mathrm{mg} / \mathrm{kg}$ body weight. ProTox predictions show no toxic 
$\mathrm{b}$
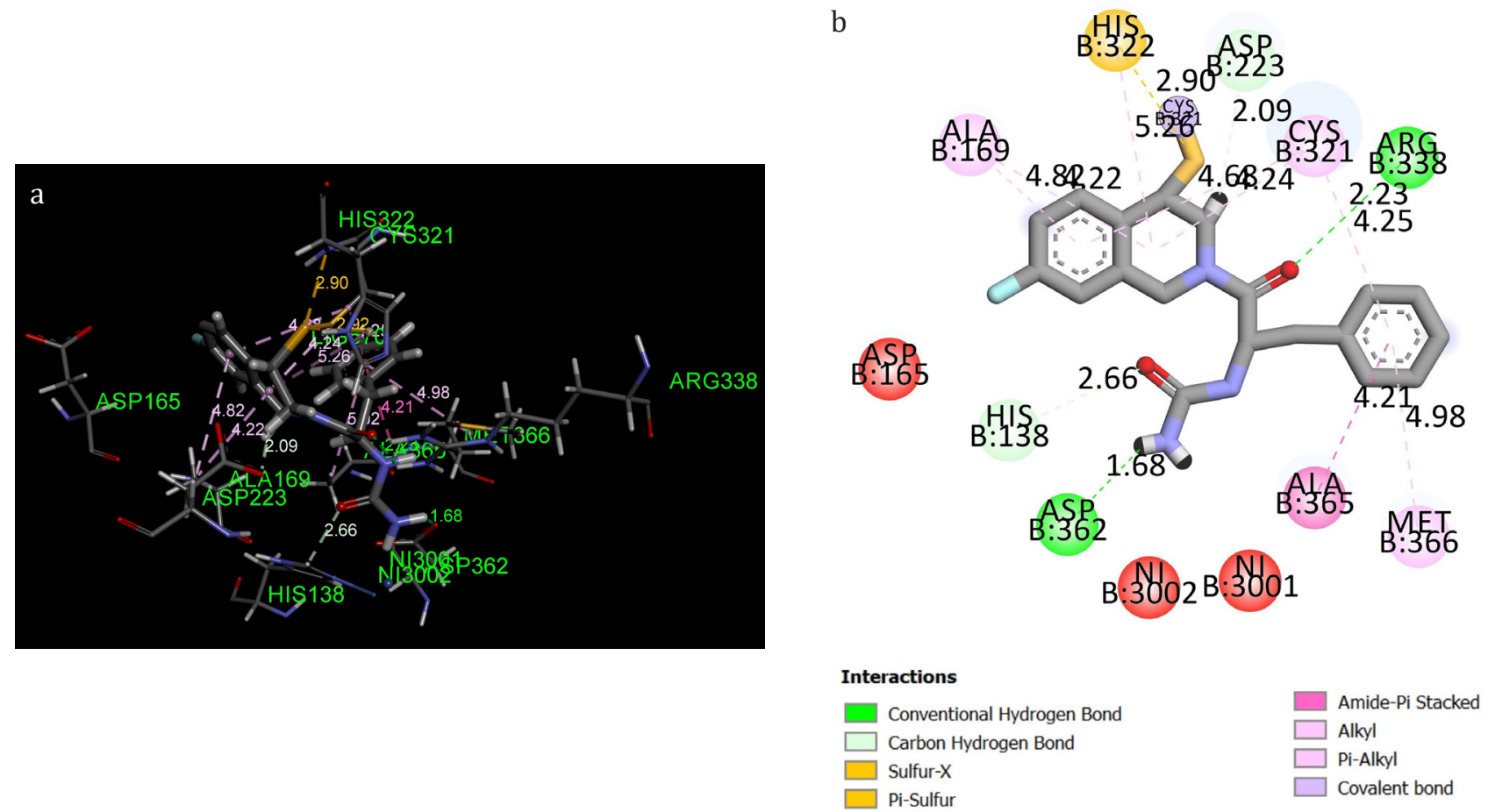

Amide-Pi Stacked

Alkyl

Pi-Alkyl

Covalent bond

Fig. 4: Covalent binding of lead derivative 1-\{(1R)-1-benzyl-2-[(4S)-7-fluoro-4-sulfanyl-3,4-dihydroisoquinolin-2(1H)-yl]-2-oxoethyl $\}$ urea to the active site of the enzyme $1 \mathrm{e} 9 \mathrm{yB}$ showing disulphide bond formation with Cys321, in 3D with atomic details

(a) and 2D at residue level only

(b) Biovia Discovery Studio 2016 visualizer has been used to prepare the figures.
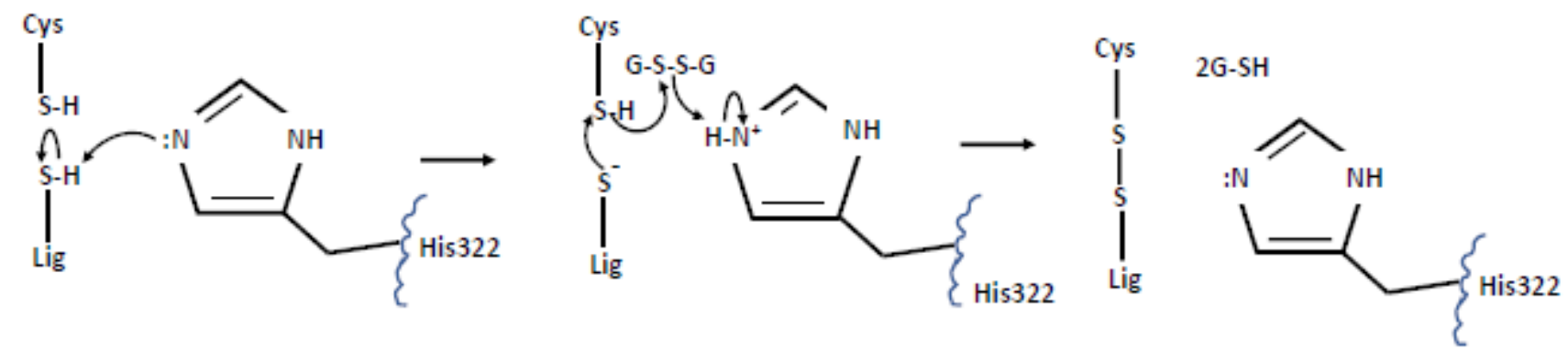

Fig. 5: Proposed mechanism showing His322 mediated nucleophilic attack on SG of Cys321 by sulfanyl group to form disulphide bond in presence of oxidizing agent namely, glutathione disulfide

reactions. It is a urea derivative. A standard approach in drug design is to use a non-active substrate derivative as a successful inhibitor due to pharmacophoric similarity. The present lead is a urea derivative, but still a novel candidate as it has not been reported as urease inhibitor to the best of our knowledge.

\section{A Covalently Bound Inhibitor}

A lead derivative 1-\{(1R)-1-benzyl-2-[(4S)-7-fluoro-4sulfanyl-3,4-dihydroisoquinolin-2(1H)-yl]-2-oxoethyl\} urea, has a potency to covalently bind to the active site through disulfide bond with Cys321 (Fig. 4a, b). The sulfanyl group is a neighbor to His322 (S12 - NE2: $3.30 \AA$ ). His322 may catalyse the formation of a disulfide bond by a mechanism proposed earlier. ${ }^{[24]}$ The NE2 of His322 may act as a base and accept hydrogen from the sulfanyl group forming a negatively charged sulfide group. Nucleophilic attack on SG of Cys 321 results in a disulfide bond. Removal of hydride in the presence of ambient oxidant in biosystems, namely, glutathione, hydrogen peroxide, has been depicted in Fig. 5. Designing covalently bound inhibitors are getting increasing attention due to their high potency, low toxicity, and increased stability of the drug-enzyme complex. ${ }^{[25-26]}$ 


\section{K. S. Chopdar et al.}

\section{REFERENCES}

1. Marshall BJ, Armstrong JA, McGechie DB, Glancy RJ. Attempt to fulfil Koch's postulates for pyloric Campylobacter. Med J Austr. 1985;142:436-439.

2. Warren JR, Marshall BJ. Unidentified curved bacilli on gastric epithelium in active chronic gastritis. Lancet. 1983; 1: 1273-1275

3. Parkin DM, Bray F, Ferlay J, Pisani P. Global cancer statistics, 2002. CA Cancer J Clin 2005;55:74-108.

4. Wroblewski LE, Peek Jr RM, Wilson Keith T. Helicobacter pylori and gastric Cancer factors that modulate disease risk. Clin Microbiol Rev. 2010;23(4):713.

5. Dunn BE, Cohen H. Blaser MJ. Helicobacter pylori. Clin Microbiol Rev. 1997;10:720-741.

6. Bayerdorffer E, O'morain C, Spille R, Unge P, van Zanten SV, Wrangstadh M, Burman CF. Antimicrobial susceptibility testing of Helicobacter pylori in a large multicenter trial: The MACH 2 study. Antimicrob. Agents Chemother. 1999;43:2747-2752

7. Andrews RK, Blakeley RL, Zerner B. Urease-A Ni (II) Metalloenzyme. in The Bioinorganic Chemistry of Nickel, Lancaster JR Jr, (Ed.), VCH Publishers, Inc: New York NY 10010.1989:141-166.

8. Mobley HL, Hausinger RP. Microbial ureases: significance, regulation, and molecular characterization. Microbiol Rev. 1989; 53:85-108.

9. Hausinger RP. Biochemistry of Nickel, Plenum Press, New York, 1993.

10. Amin M, Iqbal MS, Hughes RW, Khan SA, Reynolds PA, Enne VI, Rahman S, Mirza AS. Mechanochemical synthesis and in vitro anti-Helicobacter pylori and urease inhibitory activities of novel zinc(II)-famotidine complex.JEnzyme Inhib MedChem.2010;25:383389.

11. Megraud F. The challenge of Helicobacter pylori resistance to antibiotics: The comeback of bismuth-based quadruple therapy. Therap Adv Gastroenterol. 2012;5:103-109.

12. Ha NC, Oh ST, Sung JY, Cha KA, Lee MH, Oh BH. Supramolecular assembly and acid resistance of Helicobacter pylori urease. Nature Struct Mol Biol. 2001;8:505-509.

13. Sterling T, Irwin JJ. ZINC 15 - Ligand discovery for everyone. J Chem Inf Model. 2015;55:2324-2337.
14. Li H, Leung K-S, Ballester PJ, Wong M-H. istar: A web platform for large-scale protein-ligand docking. PLoS ONE. 2014; 9(1): e85678.

15. Hopkins AL, Groom CR, Alex A. Ligand efficiency: a useful metric for lead selection. Drug Discov Today. 2014;9:430-431.

16. Shultz MD. The thermodynamic basis for the use of lipophilic efficiency (LipE) in enthalpic optimizations. Bioorg Med Chem Lett. 2013;23:5992-6000.

17. Tarcsay A, Nyiri K, Keserü GM. Impact of lipophilic efficiency on compound quality. J Med Chem. 2012;55:1252-1260.

18. Lagorce D, Sperandio O, Baell JB, Miteva MA, Villoutreix BO. FAF-Drugs3: a web server for compound property calculation and chemical library design. Nucleic Acids Res. 2015;43(W1): W200-7.

19. Baell JB. Feeling Nature's PAINS: natural products, natural product drugs and pan assay interference compounds (PAINS). J Nat Prod. 2016;79:616-628.

20. Baell JB, Holloway GA. New substructure filters for removal of pan assay interference compounds (PAINS) from screening libraries and for their exclusion in bioassays. J Med Chem. 2010; 53: 2719-2740

21. Bickerton GR, Paolini GV, Besnard J, Muresan S, Hopkins AL. Quantifying the chemical beauty of drugs. Nat Chem. 2012;4(2): 90-98.

22. Banerjee P, Eckert AO, Schrey AK, Preissner R. ProTox-II: a webserver for the prediction of toxicity of chemicals. Nucleic Acids Res. 2018; 46:W257-W263.

23. Hassan STS, Sudomova M. The development of urease inhibitors: What opportunities exist for better treatment of Helicobacter pylori infection in children? Children (Basel). 2017;4: 2.

24. Garcin EB, Bornet O, Elantak L, Vita N, Pieulle L, Guerlesquin F, Sebban-Kreuzer C. Structural and mechanistic insights into unusual thiol disulfide oxidoreductase. J Biol Chem. 2012;287(3):1688-1697.

25. Yu HS, Gao C, Lupyan D, Wu Y, Kimura T, Wu C, Jacobson L, Harder E, Abel R, Wang L. Toward atomistic modelling of irreversible covalent inhibitor binding kinetics. J Chem Inf Model. 2019;59(9):3955-3967.

26. Zhang $\mathrm{H}$, Jiang $\mathrm{W}$, Chatterjee $\mathrm{P}$, Luo Y. Ranking reversible ccovalent drugs: From free energy perturbation to fragment docking. J Chem Inf Model. 2019;59(5):2093-2102.

HOW TO CITE THIS ARTICLE: Chopdar KS, Mohapatra PK, Nayak B, Raval MK. In silico Screening of ZINC Database for Discovery of Novel Urease Inhibitors as a remedy to gastro-duodenal ulcer caused by Helicobacter pylori. Int. J. Pharm. Sci. Drug Res. 2020;12(1):46-52. DOI: 10.25004/ IJPSDR.2020.120108 